\title{
Editorial
}

\section{Resuscitating supplementary information: is the solution a two-for-one offer?}

\author{
RA Knight ${ }^{\star, 1}$ and G Melino $0^{1,2}$ \\ Cell Death and Differentiation (2012) 19, 185; doi:10.1038/cdd.2011.177
}

More and more journals relegate scientific data into Supplementary Information, which are often not seen by the scientific community. The amount of this 'dead' data is often impressive, and papers may contain up to 30 Supplementary Figures of really high quality. Here we propose a solution by publishing them as a 'Companion' paper.

Conventional journals, such as Cell Death \& Differentiation, operate within the constraints of a page budget, generally agreed on an annual basis between the Editor-in-Chief and the Publishers, although the limitations are not so tight with journals that are completely web-based, such as Cell Death \& Disease. For conventional journals, therefore, this means that if the page budget is set at, say, 1000, then 200 papers of 5 pages can be published in one volume, but only 100 papers if their average length is 10 pages. It is obviously advantageous for the journal and for the scientific community, it serves, to publish more rather than fewer papers, and it is for this reason that there has developed greater reliance on including data that is not central to the message of the paper as Supplementary Information online. Indeed, of the 129 original papers published to date in Volume 18 (2011) of Cell Death \& Differentiation, 108 (83.7\%) included Supplementary Information of some sort.

One problem in publishing Supplementary Information is whether readers, and indeed Editors and referees, bother to access it even though it is just another click on the webpage. Anecdotally, at the journal clubs we attend, group members will have downloaded the PDF of the main text, but not the Supplementary Information. Often, of course, Supplementary Information is just that; it supplements and even complements the data in the main text. There are times, however, when the Supplementary Information is sufficiently comprehensive to add another dimension to the story and would really justify the publication as a separate paper.

A good example of the latter situation is the paper by Cesca et al. ${ }^{1}$ Here, the authors have investigated the role of kinase $D$ interacting substrate of $220 \mathrm{kDa}$ (Kidins 220; also known as ankyrin repeat-rich membrane spanning, ARMS), a membrane-bound scaffold protein for the neurotrophin receptor Trk and $\mathrm{p} 75^{\mathrm{NTR}}$ signalling. To do this, they generated Kidins $220^{-/-}$ mice and, obviously, a large amount of the data describes the generation of the mice and their characterisation. They demonstrate that the knockout mice die at a late embryonic stage with defects in the central nervous system (CNS) due to apoptosis of neurons. Moreover, primary neurons show reduced responsiveness to BDNF as assessed by the lower complexity of neurite outgrowth and by electrophysiological abnormalities. Kidins 220 was also shown to constitutively interact with VEGFR2. In the original submission, data was also included, as Supplementary Information, showing that the CNS abnormalities were accompanied by defects in the peripheral nervous system (PNS) and in the heart. In the PNS, as in the CNS, cell death occurs by apoptosis, and the cardiac phenotype includes defects in the outflow tract and in the ventricular wall. Finally, neuronal specific deletion of Kidins 220 allows live births, but the animals die early in postnatal life with profound CNS abnormalities. Because of the wealth of novel information they contain, these Supplementary Information have now been reformatted as an independent companion manuscript. $^{2}$

Clearly, in this case, the original Supplementary Information require a full exposure to the scientific community as a selfstanding publication, ${ }^{2}$ without detracting from the main paper. ${ }^{1}$ Could this be the solution to the exploding requests by editors and reviewers to add Supplementary Information to the majority of papers, and thus make more visible data which is often of considerable interest in its own right?

\section{Conflict of Interest}

The authors declare no conflict of interest.

1. Cesca F et al. Cell Death Differ 2012; 19: 194-208.

2. Cesca F et al. Cell Death Dis 2011; e-pub ahead of print 3 November 2011; doi:10.1038/ cddis.2011.108.

\footnotetext{
${ }^{1}$ MRC Toxicology Unit, Leicester LE1 9HN, UK and ${ }^{2}$ IDI-IRCCS, Department of Experimental Medicine, University of Rome Tor Vergata, Rome, Italy *Corresponding author: RA Knight, The Rayne Institute, University College London, 5, University Street, London WC1E 6JF, UK. Tel: + 44 207 9052216 ; Fax: + 44207905 2301; E-mail: r.knight@ich.ucl.ac.uk
} 